\title{
Protein tyrosine kinase 7-knockdown inhibits oral squamous cell carcinoma cell viability, proliferation, migration and invasion via downregulating dishevelled segment polarity protein 3 expression
}

\author{
XIAOYE JIN $^{1 *}$, TAO HUANG $^{2 *}$, CAIHONG MA $^{3}$, JIAFENG DUAN $^{4}$, RONG LI $^{3}$, WEI ZHANG $^{5}$ and WENYAN TIAN ${ }^{3}$ \\ ${ }^{1}$ Department of Stomatology; ${ }^{2}$ Disinfection Supply Center, The Second Hospital of Yulin City, \\ Xi'an Jiaotong University Medical School, Yulin, Shaanxi 719000; ${ }^{3}$ Department of Oral and Maxillofacial Surgery, \\ The 940th Hospital of Joint Logistics Support Force of PLA, Lanzhou, Gansu 730050; ${ }^{4}$ Department of Head and \\ Neck Cancer Surgery, Stomatological Hospital affiliated to Xi'an Jiaotong University, Xi'an, Shaanxi 710004; \\ ${ }^{5}$ Department of Pharmacy, Yulin First Hospital of Shaanxi Province, Yulin, Shaanxi 719000, P.R. China
}

Received January 29, 2021; Accepted June 11, 2021

DOI: $10.3892 / \mathrm{etm} .2021 .10806$

\begin{abstract}
Protein tyrosine kinase 7 (PTK7) expression has been reported to be dysregulated and to regulate various cellular activities in numerous types of cancer. However, to the best of our knowledge, the status and role of PTK7 in oral squamous cell carcinoma (OSCC) remains largely unknown. The present study aimed to investigate the involvement of PTK7 in OSCC progression and to determine the potential underlying mechanisms of action. The expression levels of PTK7 and dishevelled segment polarity protein 3 (DVL3) in OSCC cell lines were analyzed using reverse transcription-quantitative PCR and western blotting. A co-immunoprecipitation assay was used to verify the binding association between PTK7 and DVL3. In addition, OSCC cells were transfected with a short hairpin RNA targeting PTK7 or pcDNA-DVL3 overexpression vectors. The effect of PTK7 on OSCC cell viability, proliferation, migration and invasion, and the underlying mechanisms, were investigated using Cell Counting Kit-8, colony formation, wound healing and Transwell assays, respectively. Western blotting was used to analyze the expression levels of proliferation- and migration-associated proteins. The results revealed that the expression levels of both
\end{abstract}

Correspondence to: Dr Wenyan Tian, Department of Oral and Maxillofacial Surgery, The 940th Hospital of Joint Logistics Support Force of PLA, 333 Nanbinhe Road, Qilihe, Lanzhou, Gansu 730050, P.R. China

E-mail: tianwenlitwl@163.com

${ }^{*}$ Contributed equally

Key words: protein tyrosine kinase 7, dishevelled segment polarity protein 3 , oral squamous cell carcinoma, proliferation, migration, invasion
PTK7 and DVL3 were significantly upregulated in OSCC cell lines. In addition, a binding association was identified between PTK7 and DVL3 in SCC-9 cells. The knockdown of PTK7 expression inhibited OSCC cell viability, proliferation, invasion and migration, while the overexpression of DVL3 reversed the inhibitory effects of PTK7-knockdown on OSCC cells. In conclusion, the results of the present study suggested that PTK7 may be a key regulator of OSCC proliferation, migration and invasion, and PTK7-knockdown may inhibit OSCC cell viability, proliferation, invasion and migration by downregulating DVL3 expression. Therefore, PTK7 and DVL3 may represent potential biomarkers for diagnosis and treatment, as well as promising drug targets for OSCC.

\section{Introduction}

Oral squamous cell carcinoma (OSCC) accounts for $90 \%$ of oral cancer cases and has become a major global public health problem, as the eighth most common type of cancer worldwide (1). Due to its highly recurrent and heterogeneous nature, OSCC commonly occurs in the oral epithelium and is characterized by an insidious onset, a difficult diagnosis and rapid progression (2). In total, 300,000 new cases of OSCC are diagnosed worldwide each year, and the prevalence has significantly increased in recent years, particularly amongst the younger population (3). Risk factors for OSCC are usually lifestyle-associated and include smoking, excessive alcohol consumption and betel nut chewing (4). Although OSCC is superficial, due to the fact that individuals do not show indicative symptoms at the early stages, the diagnosis of the disease largely occurs at an extremely late stage (stage III or IV), resulting in a poor prognosis for patients, ineffective treatment and an increased social burden (5). A previous study has reported a higher rate of pathogenic tumor node metastasis in stage IV patients with OSCC, which was accompanied with an overall survival rate of $41.2 \%$, compared with in patients with stage I and II disease (6). In addition, the 5-year survival 
rate of patients with OSCC was found to be substantially decreased (7). Another study has revealed that the 5-year survival rate of patients with OSCC may be up to $90 \%$ if the disease is diagnosed early and treated appropriately (8). The high morbidity and mortality rates of OSCC highlight the urgency of identifying effective biomarkers for the early detection of OSCC.

Analysis of the Gene Expression Omnibus (GEO) dataset, GSE138206, has demonstrated that the expression levels of protein tyrosine kinase 7 (PTK7) are markedly upregulated in OSCC tissues compared with in normal tissues (9). PTK7, which is also known as colon cancer kinase 4, belongs to the receptor tyrosine kinase family and is an evolutionary, highly conserved cell surface planar cell polarity receptor $(10,11)$. Previous studies have demonstrated that PTK7 serves an extensive role in tissue development and homeostasis in vivo, affecting various aspects of communication and migration between cells $(12,13)$. Furthermore, PTK7 can control tissue morphogenesis and pattern formation by affecting cell polarity, migration and tissue regeneration and wound healing (14-16). In numerous previous studies, the dysregulation of PTK7 expression has been found to be closely associated with cancer development and with a number of cellular processes, including cell proliferation, migration and angiogenesis (17-19). Existing preclinical data have demonstrated that antibody-drug complexes targeting PTK7 may exert anti-angiogenic and immune cell-stimulating antitumor effects, thereby improving the long-term survival of patients with numerous types of cancer (20). Although the role of PTK7 in numerous human malignancies, including thyroid (21), cervical (22), adrenocortical (23) and colorectal cancer (24), has been reported, the role of PTK7 in OSCC has not yet been determined.

Dishevelled segment polarity protein (DVL)3 is a member of the dishevelled protein family, and acts as a cytoplasmic scaffold protein that links receptors to their downstream targets (25). A previous study has reported that DVL3-knockdown suppresses breast cancer cell proliferation by mediating Wnt/ $\beta$-catenin signaling (26). In esophageal squamous cell carcinoma, DVL3 downregulation can inhibit the proliferation and promote apoptosis of tumor cells (27). A meta-analysis has revealed that the DVL3 gene, which is involved in the Notch signaling pathway, is upregulated in OSCC samples, and the inhibition of Notch signaling by $\gamma$-secretase inhibitors markedly decrease the proliferation of OSCC cells in vitro (28). However, to the best of our knowledge, the biological function and clinical significance of DVL3 in OSCC has not been reported. Therefore, the present study aimed to investigate the biological role of PTK7 in OSCC and to determine its underlying mechanisms of action.

\section{Materials and methods}

Cell lines and culture. Healthy human oral keratinocytes (HOKs) were bought from ScienCell Research Laboratories, Inc. (cat. no. 2610) and OSCC cell lines (CAL-27, HSC-4 and SCC-9) were purchased from The Cell Bank of Type Culture Collection of The Chinese Academy of Sciences. Cells were cultured in DMEM (Invitrogen; Thermo Fisher Scientific, Inc.) supplemented with 10\% FBS (Invitrogen; Thermo Fisher
Scientific, Inc.) and $1 \%$ penicillin-streptomycin in a humidified incubator at $37^{\circ} \mathrm{C}$ with $5 \% \mathrm{CO}_{2}$.

Cell transfection. Short hairpin (sh) RNAs targeting PTK7 (sh-PTK7-1 and sh-PTK7-2) were used to silence PTK7 expression, and a scrambled shRNA was used as a negative control (NC) shRNA. Overexpression plasmids for DVL3 (pcDNA 3.1-DVL3) were synthesized to overexpress DVL3, and pcDNA 3.1 empty vector was used as a NC. sh-PTK7-1 $(1 \mu \mathrm{g})$, sh-PTK7-2 $(1 \mu \mathrm{g})$, NC shRNA $(1 \mu \mathrm{g})$, pcDNA-DVL3 $(50 \mathrm{nM})$ and pcDNA $(50 \mathrm{nM})$ were all designed by Shanghai GenePharma Co., Ltd. SCC-9 cells were seeded into 6-well plates $\left(1 \times 10^{6} /\right.$ well), and then transfection was performed for $8 \mathrm{~h}$ at $37^{\circ} \mathrm{C}$ using Lipofectamine ${ }^{\circledR} 3000$ (Invitrogen; Thermo Fisher Scientific, Inc.) according to the manufacturer's protocol. Cells were maintained under normal culture conditions and were harvested for analysis $72 \mathrm{~h}$ after transfection.

Reverse transcription-quantitative PCR (RT-qPCR). Total RNA was extracted from OSCC cells using TRIzol ${ }^{\circledR}$ reagent (Invitrogen; Thermo Fisher Scientific, Inc.). Total RNA was reverse-transcribed into cDNA using a PrimeScript ${ }^{\mathrm{TM}}$ RT Master Mix kit (Takara Bio, Inc.) according to the manufacturer's protocol. qPCR was subsequently performed using a TaqMan assay (Thermo Fisher Scientific, Inc.) according to the manufacturer's protocol to analyze the mRNA expression levels of PTK7 and DVL3. The following thermocycling conditions were used: Initial denaturation at $95^{\circ} \mathrm{C}$ for $10 \mathrm{~min}$; followed by 35 cycles of denaturation at $95^{\circ} \mathrm{C}$ for $15 \mathrm{sec}$, annealing at $60^{\circ} \mathrm{C}$ for $1 \mathrm{~min}$ and extension of $10 \mathrm{~min}$ at $65^{\circ} \mathrm{C}$. The following primers were used for qPCR: PTK7 forward, 5'-CAGTTCCTGAGGATTTCCAAGAG-3' and reverse, 5'-TGCATAGGGCCACCTTC-3'; DVL3 forward, 5'-CGC AAGTATGCCAGCAAC-3' and reverse, 5'-GCAGAGGTC ACCGAAGAT-3'; and GAPDH forward, 5'-GGAGCGAGA TCCCTCCAAAAT-3' and reverse, 5'-GGCTGTTGTCAT ACTTCTCATGG-3'. The expression levels were quantified using the $2^{-\triangle \Delta C q}$ method (29) and normalized to GAPDH.

Western blotting. OSCC cells were collected and lysed in RIPA lysis buffer (Beyotime Institute of Biotechnology) on ice to obtain the precipitate. Protein concentration was determined using a BCA protein assay kit (EMD Millipore) and equal amounts of protein ( $35 \mu \mathrm{g} /$ lane) were separated via $12 \%$ SDS-PAGE. The separated proteins were subsequently transferred onto PVDF membranes (EMD Millipore) and blocked at room temperature with 5\% skimmed milk for $1 \mathrm{~h}$. The membranes were then incubated with the following primary antibodies diluted in TBS-0.1\% Tween-20 (TBST) overnight at $4^{\circ} \mathrm{C}$ : Anti-PTK7 (1:200; sc-100304), anti-DVL3 (1:100; sc-271295), anti-Ki67 (1:200; sc-23900), anti-proliferating cell nuclear antigen (PCNA; 1:200; sc-56), anti-MMP2 (1:200; sc-13594), anti-MMP9 (1:100; sc-393859) and anti-GAPDH (1:200; sc-32233) (all Santa Cruz Biotechnology, Inc.). Following the primary antibody incubation, the membranes were washed thrice with TBST for $10 \mathrm{~min}$ each and incubated with HRP-conjugated goat anti-rabbit $\operatorname{IgG}$ (1:5,000; cat. no. SA00001-9) or goat anti-mouse IgG secondary antibodies (1:5,000; cat. no. SA00001-8) (both from ProteinTech Group, Inc.) at room temperature for $2 \mathrm{~h}$. Protein bands were visualized 
A

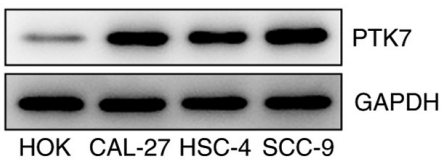

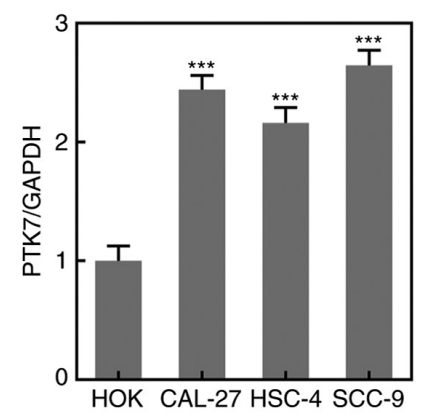

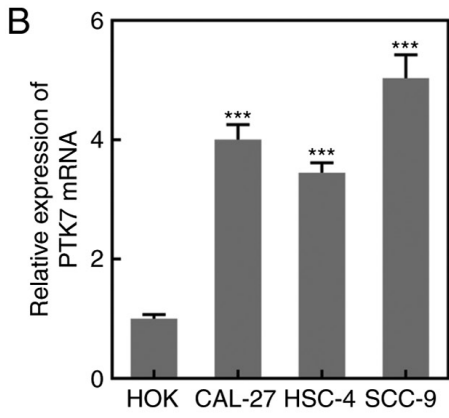

Figure 1. PTK7 expression is upregulated in OSCC cell lines. PTK7 expression in OSCC cell lines was analyzed using (A) western blotting and (B) reverse transcription-quantitative PCR. ${ }^{* * *} \mathrm{P}<0.001$ vs. HOKs. PTK7, protein tyrosine kinase 7; OSCC, oral squamous cell carcinoma; HOKs, human oral keratinocytes.

using a luminol reagent (Santa Cruz Biotechnology, Inc.) and analyzed using ImageJ software (version 1.48; National Institutes of Health).

Cell Counting Kit-8 (CCK-8) assay. SCC-9 cells transfected with NC shRNA, sh-PTK7, pcDNA or pcDNA-DVL3 were cultured in 96 -well plates at $37^{\circ} \mathrm{C}$ in $5 \% \mathrm{CO}_{2}$. Following $24 \mathrm{~h}$ of incubation, $10 \mu \mathrm{l} \mathrm{CCK}-8$ solution (Dojindo Molecular Technologies, Inc.) was added to each well and further incubated for $1 \mathrm{~h}$ at $37^{\circ} \mathrm{C}$ with $5 \% \mathrm{CO}_{2}$. The absorbance was measured at a wavelength of $450 \mathrm{~nm}$ using a microplate reader (Bio-Rad Laboratories, Inc.).

Colony formation assay. Cell proliferation was determined using a colony formation assay. Briefly, SCC-9 cells transfected with NC shRNA, sh-PTK7, pcDNA or pcDNA-DVL3 were plated into 6-well plates (with 3 replicates per condition). Following 14 days of incubation, the cells were washed with PBS, fixed with methanol for $10 \mathrm{~min}$ at room temperature and stained with $0.1 \%$ crystal violet solution for $15 \mathrm{~min}$ at room temperature (Sigma-Aldrich; Merck KGaA). The number of positively stained cells was counted.

Wound healing assay. Cell migration was analyzed using a wound healing assay. Briefly, transfected cells were seeded into 6-well plates and serum-free medium replaced normal medium. The artificial wounds were created in the cell monolayer by a single scratch with a $100-\mu \mathrm{l}$ pipette tip $(0 \mathrm{~h})$. Following $24 \mathrm{~h}$ of incubation, the wound closure area was visualized using a light microscope (Olympus Corporation) (magnification, $\mathrm{x} 100)$ and the cell migration rate was calculated using ImageJ (1.52r; National Institutes of Health).

Transwell invasion assay. A Transwell assay was used to determine cell invasion. The upper chambers of Transwell plates (Corning, Inc.) were precoated with Matrigel (BD Biosciences) overnight at $37^{\circ} \mathrm{C}$. Both transfected and non-transfected cells were seeded into the upper chamber of each well in serum-free medium (Thermo Fisher Scientific, Inc.), while $600 \mu \mathrm{l}$ medium supplemented with $10 \%$ FBS was plated into the lower chambers. Following incubation at $37^{\circ} \mathrm{C}$ with $5 \% \mathrm{CO}_{2}$ for $24 \mathrm{~h}$, non-invasive cells in the upper chamber were removed with a cotton swab, while invasive cells in the lower chambers were fixed with $4 \%$ paraformaldehyde for $30 \mathrm{~min}$ at room temperature and stained with $0.1 \%$ crystal violet solution for $20 \mathrm{~min}$ at room temperature. The invasive cells were visualized using a light microscope (Olympus Corporation; magnification, x100).

Bioinformatics analysis. Search Tool for Recurring Instances of Neighboring Genes (STRING) database 11.5 (https://www. string-db.org/) was used to analyze the connection between PTK7 and DVL3.

Co-immunoprecipitation (Co-IP) assay. To determine protein interactions in SCC-9 cells, cells were collected and lysed in lysis buffer [50 mM Tris- $\mathrm{HCl}(\mathrm{pH} 7.5), 150 \mathrm{mM} \mathrm{NaCl}, 1 \mathrm{mM}$ EDTA, $0.5 \%$ Triton X-100, $10 \%$ glycerol, $1 \mathrm{mM}$ protease inhibitor PMSF] (\#20-188; MilliporeSigma). The cell lysates (100 $\mu 1$ per antibody) were incubated with an anti-PTK7 (1 $\mu \mathrm{g}$ per $50 \mu \mathrm{g}$ total protein; \#sc-100304; Santa Cruz Biotechnology, Inc.), anti-DVL3 (1 $\mu \mathrm{g}$ per $50 \mu \mathrm{g}$ total protein, \#sc-271295; Santa Cruz Biotechnology, Inc.) or anti-IgG negative control antibody and $40 \mu 1$ protein A/G magnetic beads (\#LSKMAGAG; MilliporeSigma). The beads were subsequently washed thrice with lysis buffer and centrifuged at $500 \mathrm{x} \mathrm{g}$ for $5 \mathrm{~min}$ at $4^{\circ} \mathrm{C}$. The precipitated proteins were eluted in 1X SDS-PAGE loading buffer and boiled for $10 \mathrm{~min}$. Western blotting was performed to analyze the precipitated proteins and cell lysate, as aforementioned.

Statistical analysis. Statistical analysis was performed using GraphPad Prism 8.0 (GraphPad Software, Inc.). All experiments were repeated in triplicate and the results are presented as the mean \pm SD. Statistical differences between groups were determined using an unpaired Student's t-test or one-way ANOVA followed by Tukey's post-hoc test. $\mathrm{P}<0.05$ was considered to indicate a statistically significant difference.

\section{Results}

Expression levels of PTK7 in OSCC cell lines. To analyze the expression levels of PTK7 in OSCC cell lines, RT-qPCR and western blotting were performed. The results revealed that the protein and mRNA expression levels of PTK7 were significantly upregulated in CAL-27, HSC-4 and SCC-9 cells compared with in HOKs, with the highest expression observed in SCC-9 cells (Fig. 1A and B); therefore, SCC-9 cells were selected for use in subsequent experiments. These results 
A
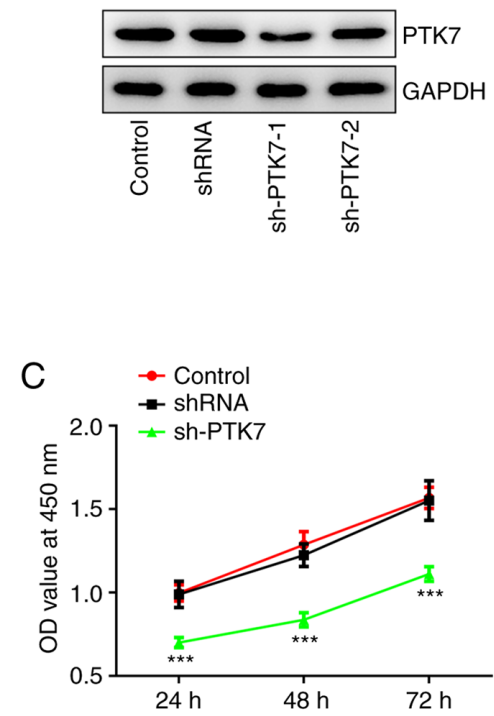

$\mathrm{E}$

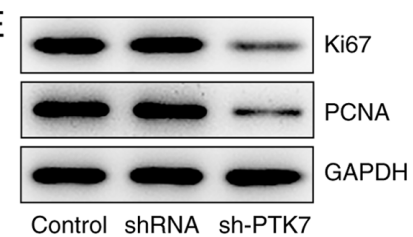

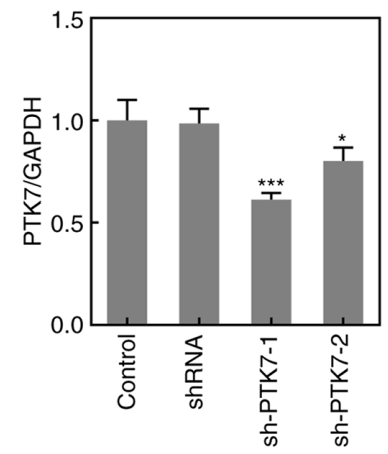
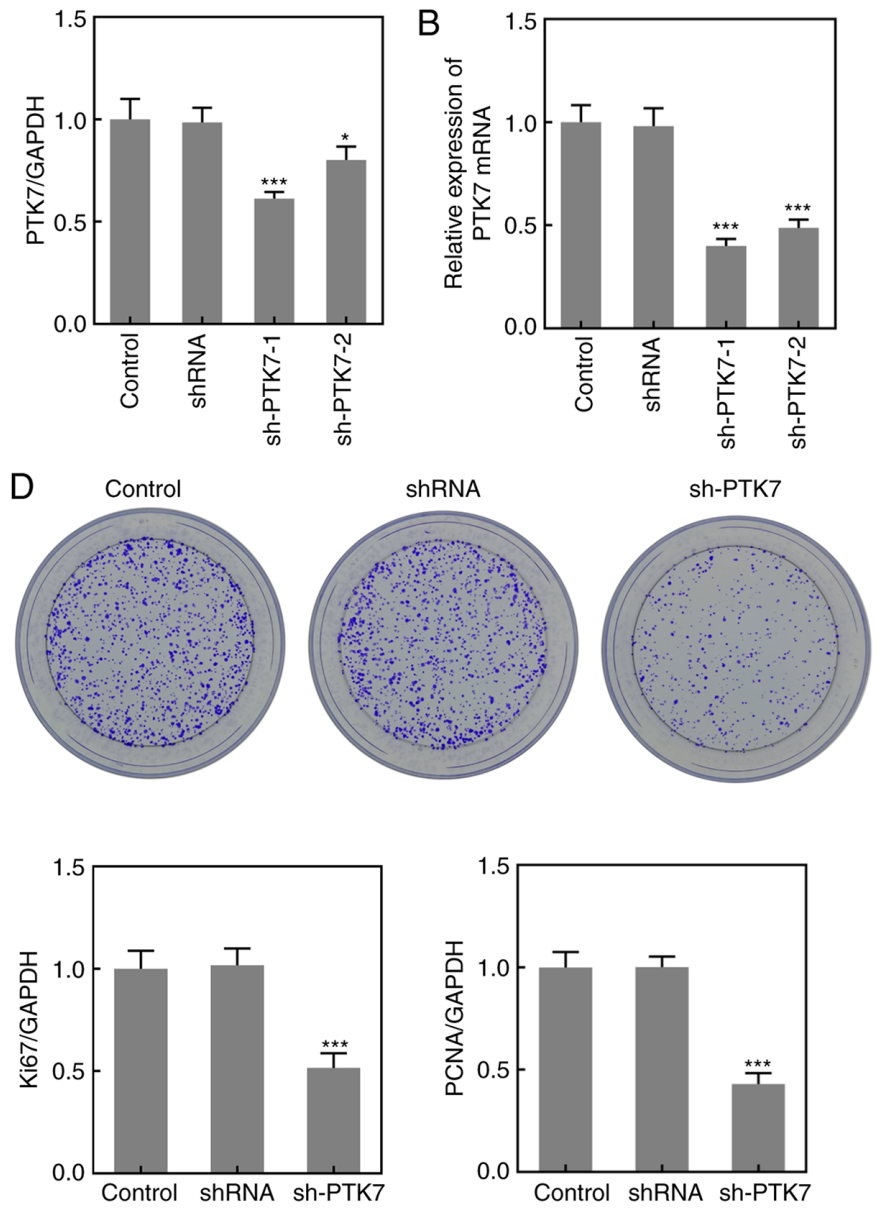

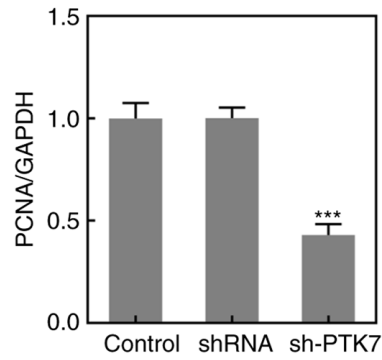

Figure 2. PTK7-knockdown inhibits SCC-9 cell viability and proliferation. PTK7 expression was measured in SCC-9 cells following transfection with sh-PTK7-1, sh-PTK7-2 or shRNA using (A) western blotting and (B) reverse transcription-quantitative PCR. (C) SCC-9 cells were transfected with sh-PTK7-1, sh-PTK7-2 or shRNA for 24, 48 or $72 \mathrm{~h}$, then cell viability was determined using a Cell Counting Kit-8 assay. (D) Proliferation of SCC-9 cells transfected with sh-PTK7-1, sh-PTK7-2 or shRNA was analyzed using a colony formation assay. (E) Ki67 and PCNA expression in SCC-9 cells following transfection with sh-PTK7-1, sh-PTK7-2 or shRNA was analyzed using western blotting. ${ }^{*} \mathrm{P}<0.05$ and ${ }^{* * * *} \mathrm{P}<0.001$ vs. shRNA. PTK7, protein tyrosine kinase 7; sh/shRNA, short hairpin RNA; PCNA, proliferating cell nuclear antigen; OD, optical density.

suggested that the expression levels of PTK7 were upregulated in OSCC cell lines.

Effect of PTK7 on OSCC cell viability and proliferation. To investigate the effect of PTK7 on OSCC cell viability and proliferation, sh-PTK7 was used to knock down PTK7 expression. As shown in Fig. 2A, both sh-PTK7-1 and sh-PTK7-2 significantly downregulated the protein expression levels of PTK7; however, sh-PTK7-1 downregulated the expression to a greater extent than sh-PTK7-2. The results obtained from the RT-qPCR analysis were similar (Fig. 2B); thus, sh-PTK7-1 was selected to knock down PTK7 expression in subsequent experiments. Subsequently, CCK- 8 assays were conducted to analyze cell viability. Compared with the control and shRNA groups, the optical density value (measured at a wavelength of $450 \mathrm{~nm}$ ) was significantly decreased in the sh-PTK7 group at 24, 48 and $72 \mathrm{~h}$ (Fig. 2C). The results of the colony formation assay revealed that the number of colonies formed was markedly decreased following PTK7-knockdown (Fig. 2D). In addition, the expression levels of the proliferation-associated proteins, Ki67 and PCNA, were analyzed and were found to be significantly downregulated in SCC-9 cells following the transfection with sh-PTK7 compared with the control group (Fig. 2E). These data suggested that the knockdown of PTK7 expression inhibited OSCC cell proliferation.

Effect of PTK7 on OSCC cell migration and invasion. The migration and invasion of SCC-9 cells were analyzed using wound healing and Transwell assays, respectively. As presented in Fig. 3A and B, the area of the wound was larger at $24 \mathrm{~h}$ and the number of invasive cells was decreased in the sh-PTK7 group compared with the NC shRNA group, which suggested that the knockdown of PTK7 expression may inhibit SCC-9 cell migration and invasion. Moreover, the expression levels of MMP2 and MMP9 were significantly downregulated following PTK7-knockdown, which further indicated the inhibitory effect of PTK-knockdown on OSCC cell migration and invasion (Fig. 3C). These results suggested that the knockdown of PTK7 expression inhibited the migration and invasion of SCC-9 cells.

Association between PTK7 and DVL3 in OSCC. To determine the potential mechanism underlying the function of PTK7 in OSCC, the Search Tool for the Retrieval of Interacting Genes/Proteins (STRING) database was used to predict that 
A

A Control
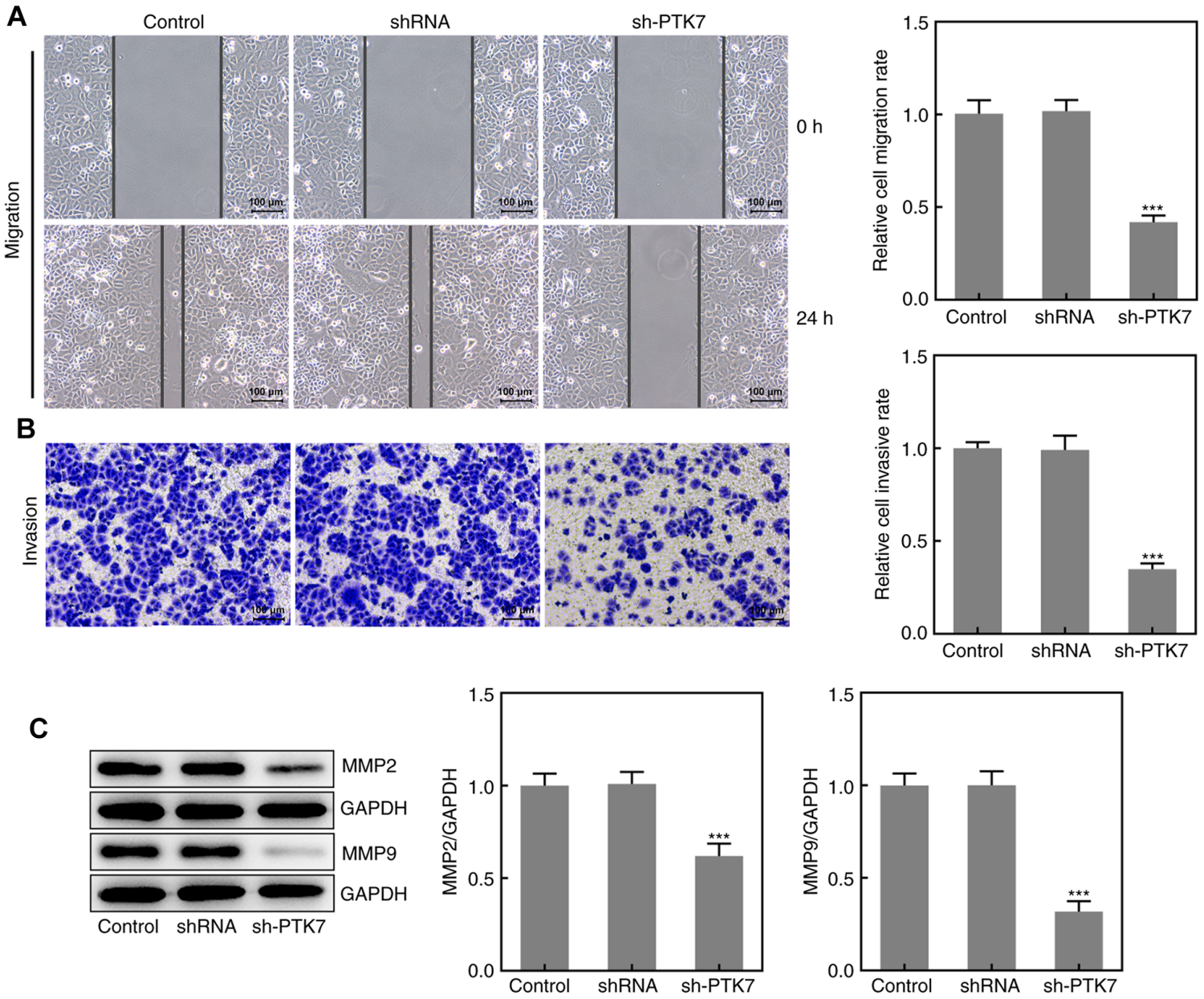

Figure 3. PTK7-knockdown inhibits SCC-9 cell migration and invasion. (A) Wound healing and (B) Transwell assays were performed to determine cell migration and invasion, respectively (magnification, x100; scale bar, $100 \mathrm{~mm}$ ). (C) MMP2 and MMP9 expression in SCC-9 cells following transfection with sh-PTK7 or shRNA was analyzed using western blotting. ${ }^{* * *} \mathrm{P}<0.001$ vs. shRNA. PTK7, protein tyrosine kinase 7 ; sh/shRNA, short hairpin RNA.

PTK7 could bind to and regulate DVL3 expression (Fig. 4F). RT-qPCR analysis and western blotting revealed that the expression levels of DVL3 were significantly upregulated in OSCC cell lines compared with in $\mathrm{HOKs}$, with the highest expression levels observed in SCC-9 cells (Fig. 4A and B). A Co-IP assay was performed to verify the binding between PTK7 and DVL3; the results revealed that DVL3 was enriched by the anti-PTK7 antibody, while PTK7 was enriched by the anti-DVL3 antibody (Fig. 4C and D). These findings indicated that PTK7 may bind to and interact with DVL3. In addition, the expression levels of DVL3 in SCC-9 cells were significantly downregulated following transfection with sh-PTK7 (Fig. 4E). These results suggested that PTK7 expression may be positively associated with DVL3 expression in OSCC.

Effect of DVL3 on OSCC cell viability and proliferation. To further determine the role of the PTK7/DVL3 interaction in regulating OSCC cell viability and proliferation, pcDNA-DVL3 was transfected into OSCC cells. The transfection efficiency of pcDNA-DVL3 was verified using western blotting (Fig. 5A). Subsequently, CCK-8 and colony formation assays were performed to determine cell viability and proliferation, respectively. The results revealed that the overexpression of DVL3 significantly alleviated the inhibitory effects of PTK7-knockdown on cell viability and colony formation (Fig. 5B and C). In addition, the expression levels of Ki67 and PCNA were significantly upregulated in the sh-PTK7 + pcDNA-DVL3 group compared with the sh-PTK7 + pcDNA group, while no significant differences were observed in the expression levels of either protein between the sh-PTK7 + pcDNA and sh-PTK7 groups (Fig. 5D). These results suggested that the overexpression of DVL3 may reverse the PTK7-knockdown-induced inhibitory effects on the proliferation and viability of OSCC cells.

Effect of DVL3 on OSCC cell migration and invasion. Wound healing and Transwell assays were performed to determine the effects of the overexpression of DVL3 on the migration and invasion of SCC-9 cells, respectively. The results demonstrated that cell migration and invasion were significantly increased following the co-transfection of sh-PTK7 and pcDNA-DVL3 compared with sh-PTK7 alone, indicating that the overexpression of DVL3 may ameliorate the inhibitory effects of sh-PTK7 (Fig. 6A and B). The protein expression levels of MMP2 and MMP9 were also significantly upregulated in the sh-PTK7 + pcDNA-DVL3 group compared 
A

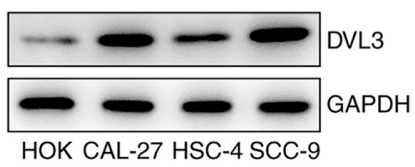

C

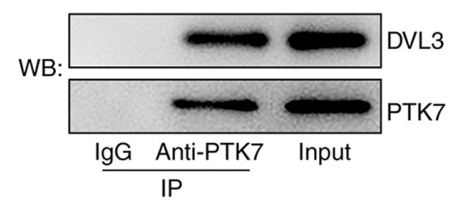

D

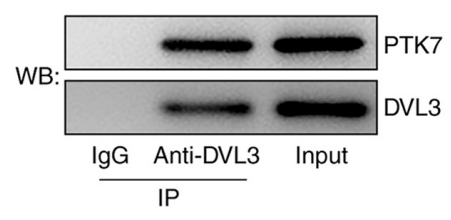

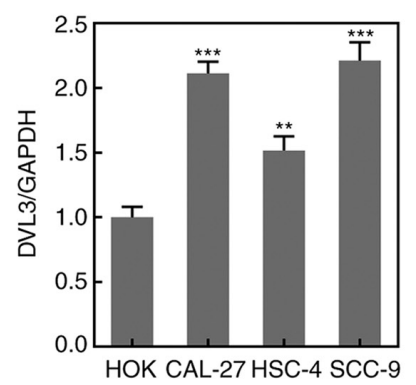
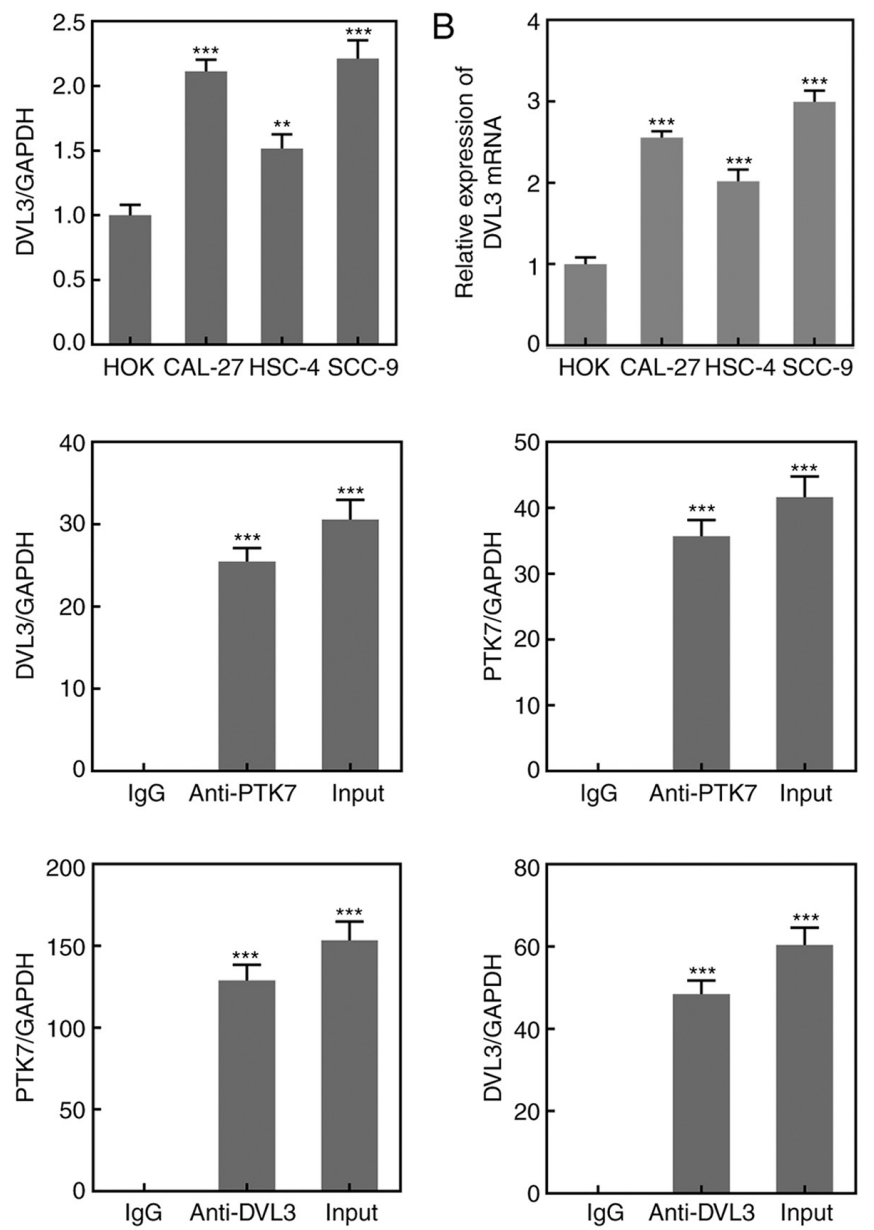

$E$

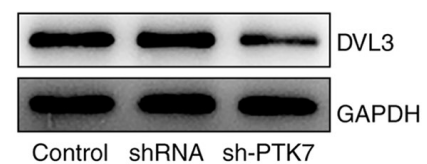

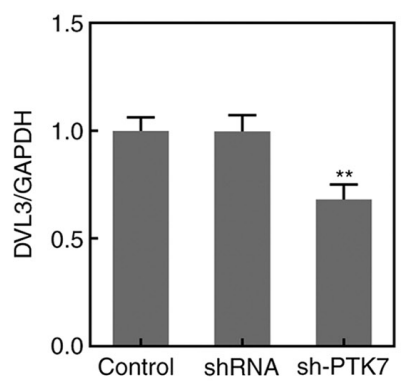

$\mathrm{F}$

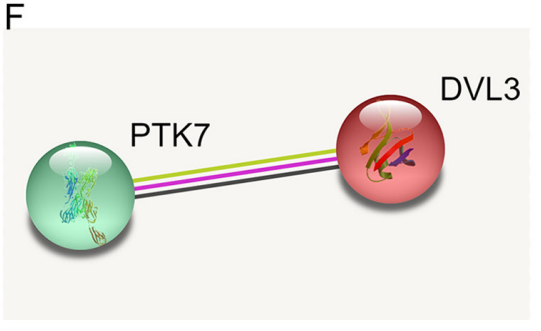

Figure 4. PTK7 expression is positively associated with DVL3 expression. The expression levels of DVL3 in oral squamous cell carcinoma cell lines were analyzed using (A) western blotting and (B) reverse transcription-quantitative PCR. ${ }^{* *} \mathrm{P}<0.01$ and ${ }^{* * *} \mathrm{P}<0.001$ vs. HOKs. (C and D) Association between PTK7 and DVL3 was analyzed using a co-immunoprecipitation assay. ${ }^{* * *} \mathrm{P}<0.001$ vs. IgG. (E) DVL3 expression in SCC-9 cells following transfection with sh-PTK7 was analyzed using western blotting. (F) The relationship between PTK7 and DVL3 was predicted using the Search Tool for the Retrieval of Interacting Genes/Proteins database. ${ }^{* *} \mathrm{P}<0.01$ vs. shRNA. PTK7, protein tyrosine kinase 7; DVL3, dishevelled segment polarity protein; HOKs, human oral keratinocytes; sh/shRNA, short hairpin RNA.

with the sh-PTK7 + pcDNA group (Fig. 6C). These results suggested that the overexpression of DVL3 may reverse the sh-PTK7-induced inhibitory effects on the migration and invasion of OSCC cells.

\section{Discussion}

The present study aimed to investigate the tumor-promoting effect of PTK7 in OSCC. The results revealed that the expression levels of PTK7 were upregulated in OSCC cell lines and that PTK7-knockdown inhibited the viability, proliferation, migration and invasion of OSCC cells. In addition, DVL3 was identified to be positively associated with PTK7, and the overexpression of DVL3 ameliorated the inhibitory effects of sh-PTK7 on these biological processes.

Numerous previous studies have reported the role of PTK7 in several types of cancer. For example, PTK7 has been identified as a potential target for cervical cancer treatment, since knocking down PTK7 expression inhibits the proliferative, migratory and invasive abilities of cervical cancer cells both in vivo and in vitro (22). Another previous study has reported that microRNA-205-5p inhibits the migration and invasion of colon cancer cells by downregulating PTK7 expression (24). PTK7 expression has been also found to be upregulated in OSCC cells compared with in normal squamous cells, and high PTK7 
A

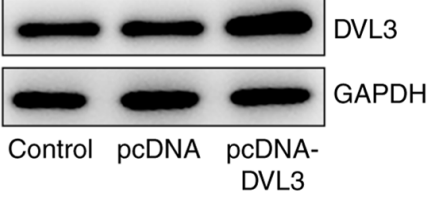

C

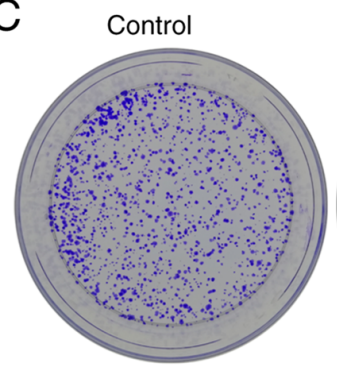

D

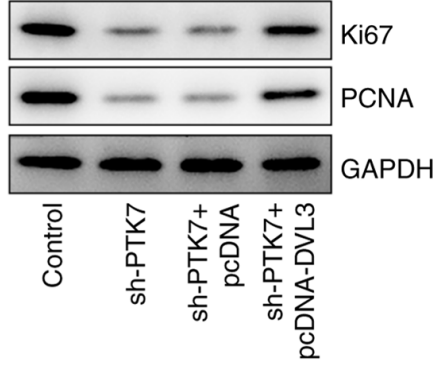

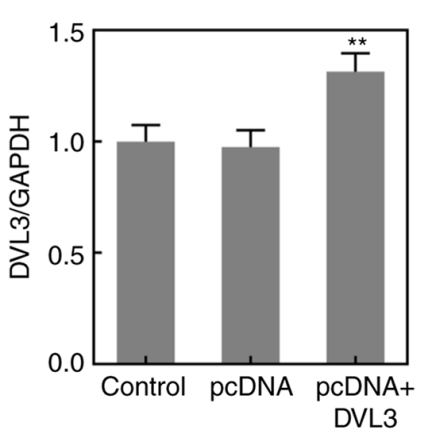

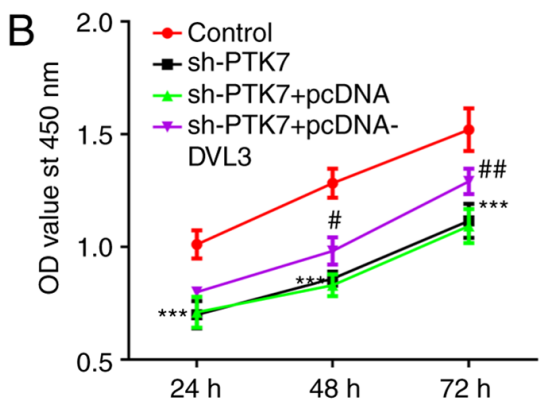

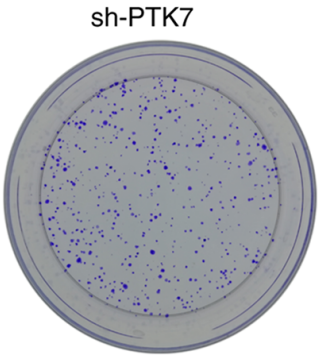

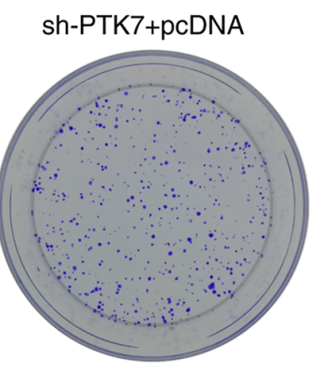

sh-PTK7+pcDNA-DVL3

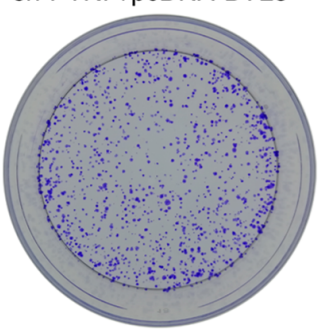

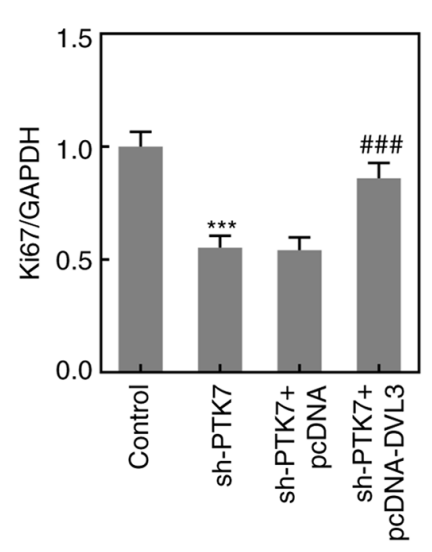

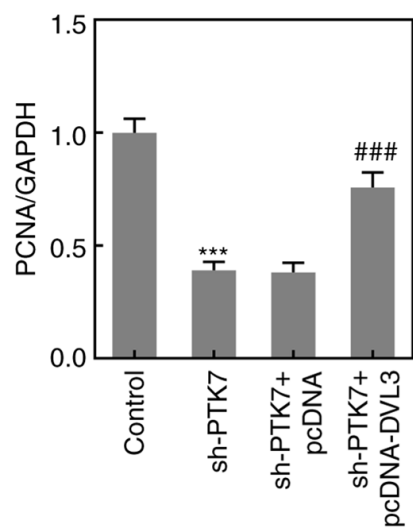

Figure 5. Overexpression of DVL3 reverses the sh-PTK7-induced inhibition of SCC-9 cell viability and proliferation. (A) DVL3 expression in SCC-9 cells following transfection with pcDNA or pcDNA-DVL3 was analyzed using western blotting. (B) SCC-9 cells were transfected with pcDNA or pcDNA-DVL3 for 24,48 or $72 \mathrm{~h}$, then cell viability was analyzed using a Cell Counting Kit-8 assay. (C) Proliferation of SCC-9 cells transfected with pcDNA or pcDNA-DVL3 was analyzed using a colony formation assay. (D) Ki67 and PCNA expression in SCC-9 cells following transfection with pcDNA or pcDNA-DVL3 was analyzed using western blotting. ${ }^{* *} \mathrm{P}<0.01$ and ${ }^{* * *} \mathrm{P}<0.001$ vs. control; ${ }^{\#} \mathrm{P}<0.05,{ }^{\# \#} \mathrm{P}<0.01$ and ${ }^{\# \#} \mathrm{P}<0.001$ vs. sh-PTK7 + pcDNA. DVL3, dishevelled segment polarity protein; sh, short hairpin RNA; PTK7, protein tyrosine kinase 7; PCNA, proliferating cell nuclear antigen; OD, optical density.

expression has been positively associated with TNM stage, tumor differentiation and lymph node metastasis. In addition, patients with higher PTK7 expression exhibit a poorer overall survival (30). These data indicate that PTK7 is associated with OSCC prognosis and may represent a potential therapeutic target (30). The present study first analyzed the expression levels of PTK7 in OSCC cell lines, and the results obtained were consistent with the observed expression levels of PTK7 in OSCC tissues from the GEO database (9), indicating that PTK7 expression may be upregulated in OSCC. Next, the potential biological role of PTK7 in OSCC cells was investigated using sh-PTK7. Cancer cell proliferation determines cancer progression, and metastasis, characterized by cancer cell migration and invasion, represents a significant challenge in the clinical treatment of various types of cancer (31). Therefore, the current study sought to determine the effect of PTK7 on OSCC cell proliferation, migration and invasion. The results demonstrated that the knockdown of PTK7 expression suppressed the viability, proliferation, migration and invasion of OSCC cells.

The potential mechanisms underlying the biological effects of PTK7 were investigated. The STRING database was used to predict that PTK7 could bind to and regulate DVL3 expression. Notably, DVL3, as a gene of the Notch signaling pathway, has been previously reported to be upregulated in OSCC samples (28). In addition, the knockdown of DVL3 expression has been found to control the progression of esophageal squamous cell carcinoma, inhibit cell proliferation and promote the apoptosis of tumor cells (27). Moreover, a recent study has revealed that testis-specific transcript, Y-linked 15 downregulates DVL3 expression in colorectal cancer tissues and promotes the proliferation, migration and invasion of colorectal cancer cells (32). The findings of the present study revealed that the expression levels of DVL3 were upregulated in OSCC cell lines, and a Co-IP assay confirmed 

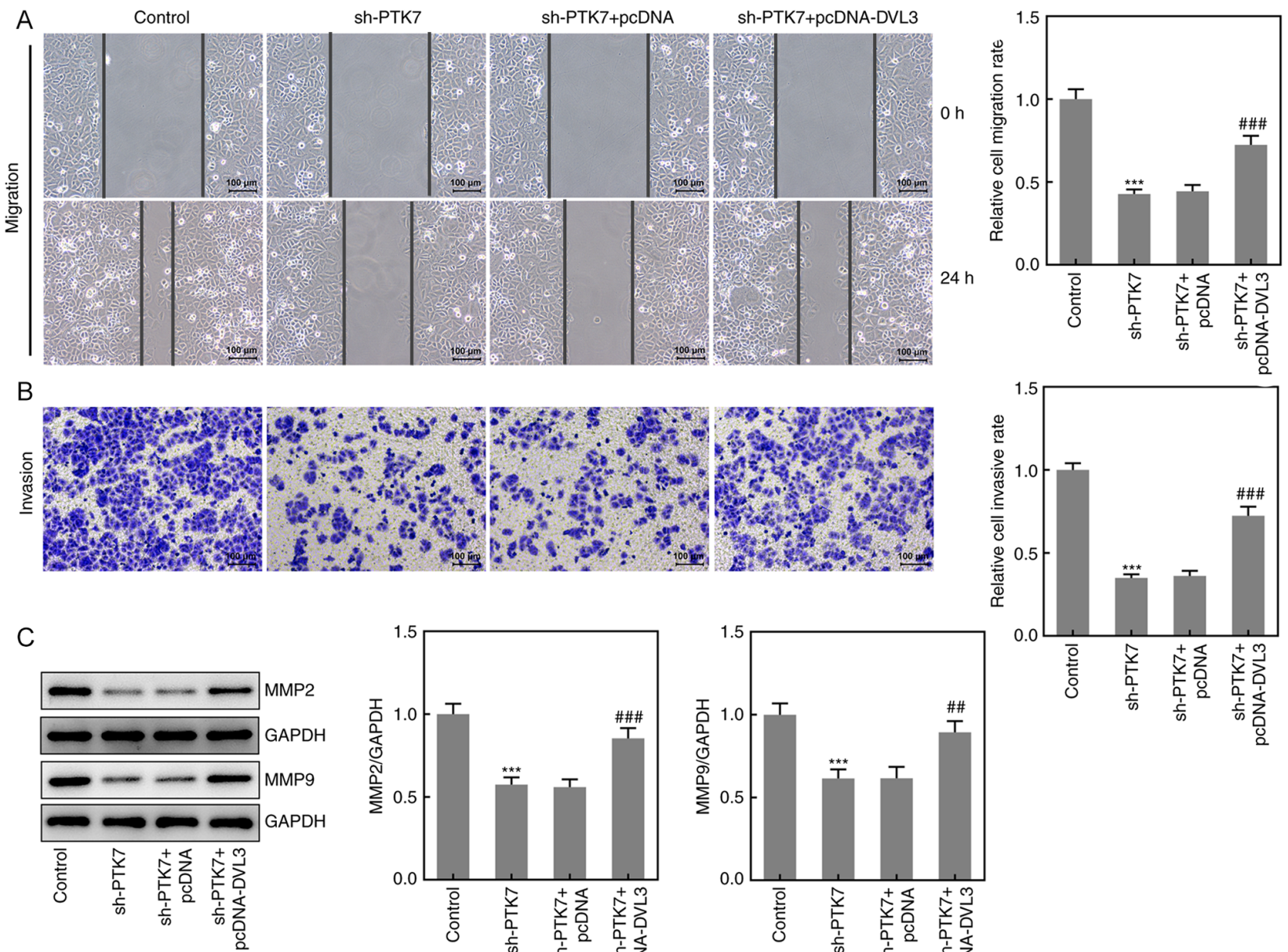

Figure 6. Overexpression of DVL3 reverses the sh-PTK7-induced inhibition of SCC-9 cell migration and invasion. (A) Wound healing and (B) Transwell assays were performed to analyze cell migration and invasion, respectively (magnification, x100; scale bar, 100 mm). (C) MMP2 and MMP9 expression in SCC-9 cells following transfection with sh-PTK7, pcDNA or pcDNA-DVL3 were analyzed using western blotting. ${ }^{* * * *} \mathrm{P}<0.001 \mathrm{vs}$. control; ${ }^{\# \# /} \mathrm{P}<0.01$ and

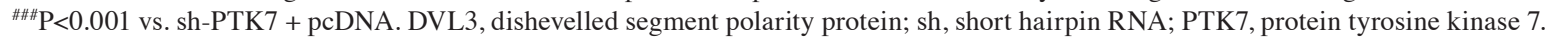

the interaction between PTK7 and DVL3. Subsequently, pcDNA-DVL3 was co-transfected with sh-PTK7 into OSCC cells, and the results demonstrated that the overexpression of DVL3 reversed the inhibitory effects of sh-PTK7 on OSCC cells. Notably, it was found that PTK7 positively associated with DVL3 expression and that inhibition of PTK7 expression inhibited the proliferation, migration and invasion of OSCC cells. A previous study has also indicated that PTK7 interacts with AMIGO2 and is able to act as a survival factor in melanoma (33). Therefore, it is possible that PTK7 and DVL3 also interact with each other in OSCC, and this should be further explored in future studies.

In conclusion, the findings of the current study revealed that the expression levels of PTK7 and DVL3 were upregulated in OSCC. PTK7-knockdown inhibited OSCC cell viability, proliferation, migration and invasion by downregulating DVL3 expression. These results may provide novel insight into the potential role of PTK7 and DVL3 in the clinical prognosis and treatment of OSCC.

\section{Acknowledgements}

Not applicable.

\section{Funding}

No funding was received.

\section{Availability of data and materials}

The datasets used and/or analyzed during the current study are available from the corresponding author on reasonable request.

\section{Authors' contributions}

$\mathrm{XJ}$ and WT conceived and designed the experiments. TH, CM and JD performed the experiments. RL and WZ analyzed the data. XJ and TH wrote the manuscript. All authors read and approved the final manuscript and confirm the authenticity of the raw data.

\section{Ethics approval and consent to participate}

Not applicable.

\section{Patient consent for publication}

Not applicable. 


\section{Competing interests}

The authors declare that they have no competing interests.

\section{References}

1. Chai AWY, Lim KP and Cheong SC: Translational genomics and recent advances in oral squamous cell carcinoma. Semin Cancer Biol 61: 71-83,2020.

2. Hübbers CU and Akgül B: HPV and cancer of the oral cavity Virulence 6: 244-248, 2015.

3. Sasahira T and Kirita T: Hallmarks of cancer related newly prognostic factors of oral squamous cell carcinoma. Int J Mol Sci 19: 2413, 2018

4. Huang SH and O'Sullivan B: Overview of the 8th Edition TNM Classification for Head and Neck Cancer. Curr Treat Options Oncol 18: 40, 2017.

5. Roi A, Roi CI, Negrutiu ML, Rivis M, Sinescu C and Rusu LC: The Challenges of OSCC diagnosis: Salivary cytokines as potential biomarkers. J Clin Med 9: 2866, 2020.

6. Campisi G, Calvino F, Carinci F, Matranga D, Carella M, Mazzotta M, Rubini C, Panzarella V, Santarelli A, Fedele S, et al: Peri-tumoral inflammatory cell infiltration in OSCC: A reliable marker of local recurrence and prognosis? An investigation using artificial neural networks. Int J Immunopathol Pharmacol 24 113-120, 2011.

7. Kreppel M, Nazarli P, Grandoch A, Safi AF, Zirk M, Nickenig HJ, Scheer M, Rothamel D, Hellmich M and Zöller JE: Clinical and histopathological staging in oral squamous cell carcinoma - comparison of the prognostic significance. Oral Oncol 60: 68-73, 2016.

8. Cristaldi M, Mauceri R, Di Fede O, Giuliana G, Campisi G and Panzarella V: Salivary biomarkers for oral squamous cell carcinoma diagnosis and follow-up: Current Status and Perspectives. Front Physiol 10: 1476, 2019.

9. Guo ZC, Jumatai S, Jing SL, Hu LL, Jia XY and Gong ZC: Bioinformatics and immunohistochemistry analyses of expression levels and clinical significance of CXCL2 and TANs in an oral squamous cell carcinoma tumor microenvironment of Prophyromonas gingivalis infection. Oncol Lett 21: 189, 2021.

10. Berger H, Wodarz A and Borchers A: PTK7 faces the Wnt in development and disease. Front Cell Dev Biol 5: 31, 2017.

11. Mossie K, Jallal B, Alves F, Sures I, Plowman GD and Ullrich A: Colon carcinoma kinase-4 defines a new subclass of the receptor tyrosine kinase family. Oncogene 11: 2179-2184, 1995.

12. Golubkov VS, Prigozhina NL, Zhang Y, Stoletov K, Lewis JD, Schwartz PE, Hoffman RM and Strongin AY: Protein-tyrosine pseudokinase 7 (PTK7) directs cancer cell motility and metastasis. J Biol Chem 289: 24238-24249, 2014

13. González P, González-Fernández C, Campos-Martín Y, Mollejo M, Carballosa-Gautam M, Marcillo A, Norenberg M, García-Ovejero D and Rodríguez FJ: Spatio-temporal and Cellular Expression Patterns of PTK7 in the Healthy and Traumatically Injured Rat and Human Spinal Cord. Cell Mol Neurobiol 40: 1087-1103, 2020.

14. Bie J, Hu X, Yang M, Shi X, Zhang X and Wang Z: PTK7 promotes the malignant properties of cancer stem-like cells in esophageal squamous cell lines. Hum Cell 33: 356-365, 2020

15. Peradziryi H, Tolwinski NS and Borchers A: The many roles of PTK7: A versatile regulator of cell-cell communication. Arch Biochem Biophys 524: 71-76, 2012.

16. Katoh M: Canonical and non-canonical WNT signaling in cancer stem cells and their niches: Cellular heterogeneity, omics reprogramming, targeted therapy and tumor plasticity (Review) Int J Oncol 51: 1357-1369, 2017.

17. Tian X, Yan L, Zhang D, Guan X, Dong B, Zhao M and Hao C: PTK7 overexpression in colorectal tumors: Clinicopathological correlation and prognosis relevance. Oncol Rep 36: 1829-1836, 2016.
18. Kadioglu O, Saeed MEM, Mahmoud N, Hussein Azawi SS, RincicM,LiehrT andEfferth T: Identification of metastasis-related genes by genomic and transcriptomic studies in murine melanoma. Life Sci 267: 118922, 2021.

19. Nweke EE, Naicker P, Aron S, Stoychev S, Devar J, Tabb DL, Omoshoro-Jones J, Smith M and Candy G: SWATH-MS based proteomic profiling of pancreatic ductal adenocarcinoma tumours reveals the interplay between the extracellular matrix and related intracellular pathways. PLoS One 15: e0240453, 2020.

20. Damelin M, Bankovich A, Bernstein J, Lucas J, Chen L, Williams S, Park A, Aguilar J, Ernstoff E, Charati M, et al: A PTK7 targeted antibody drug conjugate reduces tumor initiating cells and induces sustained tumor regressions. Sci Transl Med 9: eaag2611, 2017.

21. Duan F, Tang J, Kong FL,Zou HW, Ni BL and Yu JC: Identification of PTK7 as a promising therapeutic target for thyroid cancer. Eur Rev Med Pharmacol Sci 24: 6809-6817, 2020.

22. Sun JJ, Li HL, Guo SJ, Ma H, Liu SJ, Liu D and Xue FX: The increased PTK7 expression is a malignant factor in cervical cancer. Dis Markers 2019: 5380197, 2019.

23. Bie J, Liu K, Song G, Hu X, Xiong R, Zhang X, Shi X and Wang Z: ENST00000489707.5 is a preferred alternative splicing variant of PTK7 in adrenocortical cancer and shows potential prognostic value. Med Sci Monit 25: 8326-8334, 2019.

24. Chen S, Wang Y, Su Y, Zhang L, Zhang M, Li X, Wang J and Zhang X: miR 205 5p/PTK7 axis is involved in the proliferation, migration and invasion of colorectal cancer cells. Mol Med Rep 17: 6253-6260, 2018

25. Sharma M, Castro-Piedras I, Simmons GE Jr and Pruitt K: Dishevelled: A masterful conductor of complex Wnt signals. Cell Signal 47: 52-64, 2018.

26. Zou YF, Xie CW, Yang SX and Xiong JP: AMPK activators suppress breast cancer cell growth by inhibiting DVL3-facilitated Wnt/ $\beta$-catenin signaling pathway activity. Mol Med Rep 15: 899-907, 2017.

27. Chen XQ, Jiang J, Wang XT, Zhang CL, Ji AY and Chen XJ: Role and mechanism of Dvl3 in the esophageal squamous cell carcinoma. Eur Rev Med Pharmacol Sci 22: 7716-7725, 2018

28. Osathanon T, Nowwarote N and Pavasant P: Expression and influence of Notch signaling in oral squamous cell carcinoma. J Oral Sci 58: 283-294, 2016.

29. Livak KJ and Schmittgen TD: Analysis of relative gene expression data using real-time quantitative PCR and the 2(-Delta Delta C(T)) Method. Methods 25: 402-408, 2001

30. Dong Y, Chen X, Li H, Ni Y, Han W and Wang J: PTK7 is a molecular marker for metastasis, TNM stage, and prognosis in oral tongue squamous cell carcinoma. Pol J Pathol 68: 49-54, 2017.

31. Zuo K, Qi Y, Yuan C, Jiang L, Xu P, Hu J, Huang M and Li J: Specifically targeting cancer proliferation and metastasis processes: The development of matriptase inhibitors. Cancer Metastasis Rev 38: 507-524, 2019.

32. Zheng XY, Cao MZ, Ba Y, Li YF and Ye JL: LncRNA testis specific transcript, Y linked 15 (TTTY15) promotes proliferation, migration and invasion of colorectal cancer cells via regulating miR 29a 3p/DVL3 axis. Cancer Biomark 31: 1-11, 2021.

33. Fontanals-Cirera B, Hasson D, Vardabasso C, Di Micco R, Agrawal P, Chowdhury A, Gantz M, de Pablos-Aragoneses A, Morgenstern A, Wu P, et al: Harnessing BET inhibitor sensitivity reveals AMIGO2 as a melanoma survival gene. Mol Cell 68 : 731-744.e9, 2017.

This work is licensed under a Creative Commons Attribution-NonCommercial-NoDerivatives 4.0 International (CC BY-NC-ND 4.0) License. 